\title{
High expression of HMGA2 predicts poor survival in patients with clear cell renal cell carcinoma
}

This article was published in the following Dove Press journal:

OncoTargets and Therapy

22 November 2016

Number of times this article has been viewed

\author{
Ning $\mathrm{Na}^{1, *}$ \\ Tujie $\mathrm{Si}^{2}, *$ \\ Zhengyu Huangl,* \\ Bin Miao' \\ Liangqing Hong' \\ Heng $\mathrm{Li}^{\prime}$ \\ Jiang Qiu \\ Jianguang $\mathrm{Qiu}^{3}$ \\ 'Department of Kidney \\ Transplantation, The Third Affiliated \\ Hospital of Sun Yat-sen University, \\ ${ }^{2}$ Department of Organ Transplant, \\ The First Affiliated Hospital of Sun \\ Yat-sen University, ${ }^{3}$ Department of \\ Urology, The Third Affiliated Hospital \\ of Sun Yat-sen University, Guangzhou, \\ People's Republic of China \\ *These authors contributed equally \\ to this work
}

Correspondence: Jianguang Qiu

Department of Urology, The Third Affiliated Hospital of Sun Yat-sen

University, Guangzhou, Guangdong

Province 510630, People's Republic

of China

Tel +862085252175

Fax +86 2085253336

Email qiujianguang_sysu@|26.com

\begin{abstract}
High-mobility group AT-hook 2 (HMGA2) is involved in a wide spectrum of biological processes and is upregulated in several tumors, but its role in renal carcinoma remains unclear. The aim of this study was to examine the expression of HMGA2 and its relationship to the overall survival (OS) of patients with non-metastatic clear cell renal cell carcinoma (ccRCC) following surgery. The expression of HMGA2 was evaluated retrospectively by immunohistochemistry (IHC) in 162 patients with ccRCC who underwent nephrectomy in 2003 and 2004. An IHC analysis revealed that HMGA2 was expressed in the nuclei of tumor cells in $146(90.1 \%)$ patients with ccRCC. The level of HMGA2 was positively correlated with tumor size, lymph node metastasis, and Fuhrman Grade. A Kaplan-Meier analysis with log-rank test found that patients with high HMGA2 expression had a poor outcome and that patients with low HMGA2 expression had better survival. Cox regression analysis showed that HMGA2 expression could serve as an independent prognostic factor for ccRCC patients. The efficacy of the following prognostic models was improved when HMGA2 expression was added: tumor node metastasis stage, UCLA Integrated Scoring System, Mayo Clinic stage, size, grade, and necrosis score. In summary, this study showed that HMGA2 expression is an independent prognostic factor for OS in patients with ccRCC. HMGA2 was found to be a valuable biomarker for ccRCC progression.
\end{abstract}

Keywords: renal carcinoma, high-mobility group protein A, prognosis

\section{Introduction}

Renal cell carcinoma (RCC) is the most common urologic tumor in adults and accounts for approximately $3 \%$ of all human malignancies. ${ }^{1}$ Clear cell renal cell carcinoma (ccRCC), which is the most common histological subtype of RCC, accounts for $85 \%$ to $90 \%$ of renal malignancies and has the worst prognosis among RCCs. ${ }^{2}$ Surgery is the preferred treatment for localized ccRCC, but as many as $30 \%$ of patients who undergo surgery with curative intent experience local recurrence or distant metastasis, which often leads to a poor prognosis. ${ }^{3}$ Traditional prognostic factors, such as tumor node metastasis (TNM) stage, presence of necrosis, and ECOG-PS, as well as a combination of these factors provide a useful benchmark to determine prognosis and select a treatment strategy. However, sometimes discrepancies arise between predictions and practical clinical outcomes. ${ }^{4}$ Recent studies have revealed a number of prognostic biomarkers that are associated with different features of RCC biology, and novel models that combine conventional clinicopathological factors with molecular biomarkers have been established to allow for more a precise prediction of clinical outcomes. ${ }^{5-8}$ Therefore, it is of great value to seek molecular markers that will improve the individual therapeutic management and clinical outcome of patients with ccRCC. 
The high-mobility group A (HMGA) protein family comprises the high-mobility group AT-hook 1 (HMGA1; coded by a gene on chromosome $6 \mathrm{p} 21$ through alternative splicing) and high-mobility group AT-hook 2 (HMGA2; coded by a gene on chromosome 12q14-15) proteins, which are characterized by their ability to bind to nucleotide sites rich in adenine and thymine. ${ }^{9}$ The HMGA proteins act as architectural transcription factors by altering the conformation of transcription factors and the chromatin structure, and thus these proteins modulate gene transcription. ${ }^{10} \mathrm{HMGA}$ proteins are expressed at high levels during embryonic development, whereas their expression is strongly reduced or nearly absent in adult organs. ${ }^{11}$ The re-expression of both HMGA1 and HMGA2 at high levels in adults is seen in a variety of malignant and benign tumors. ${ }^{10,12}$ It has been generally recognized that both the HMGA1 and HMGA2 proteins play a role in malignant cell transformation and progression of several tumor types. Recent studies have revealed that HMGA1 is overexpressed in RCC and plays a key role in the control of the aggressiveness of renal cancer cells. ${ }^{12}$ However, the role of HMGA2 in renal carcinoma is still unknown.

In this study, we examined the expression of HMGA2 by IHC in ccRCC tissues derived from patients who underwent radical surgery. We then aimed to evaluate the association between HMGA2 expression and prognosis and its potential utility as a prognostic marker for ccRCC to develop a better understanding of the role of HMGA2 in ccRCC.

\section{Materials and methods}

\section{Patients and samples}

The current study included 162 patients with localized or locally advanced ccRCC who presented at The First Affiliated Hospital and The Third Affiliated Hospital of Sun Yat-sen University between 2003 and 2004. The histological type of all specimen sections was determined by experienced pathologists, and all samples were diagnosed as conventional ccRCC. The clinical tumor stages and characteristics were classified according to the TNM classification system. The original inclusion criteria were as follows: 1) patients who received radical nephrectomy by either the open approach or by laparoscopy; 2) confirmation of ccRCC by postoperative histopathologic diagnosis; 3) no radiotherapy or chemotherapy before surgery; and 4) $M=0$ based on the JCC TNM classification. Patients who died of postoperative complications were excluded. This research is in compliance with the Helsinki Declaration and was approved by the Institutional Ethics Review Committee of Sun Yat-sen University. Written informed consents were obtained from all patients involved in this study.

\section{Immunohistochemistry}

IHC was performed on paraffin-embedded samples of the primary carcinomas from 162 patients. The pathological sections were deparaffinized in xylene and rehydrated in a graded series of ethanol, followed by antigen retrieval in a microwave. The sections were incubated with $0.3 \%$ hydrogen peroxide for $10 \mathrm{~min}$ at room temperature to quench the activity of endogenous peroxidase. The sections were then incubated with a rabbit HMGA2 polyclonal antibody (Abcam; Rabbit polyclonal to HMGA2; ab52039) overnight at $4^{\circ} \mathrm{C}$ at an optimal working dilution of 1:100. After sufficient rinses in phosphate buffered saline (PBS), the sections were incubated with goat anti-rabbit polymers. The staining was scored by two independent investigators who were blinded to the clinicopathological and prognostic data. The staining intensity ( 0 : negative, 1 : weak staining, 2 : moderate staining, and 3: strong staining) and staining extent $(0-100)$ were evaluated in ten randomly selected high-power fields. A semiquantitative IHC score was generated by calculating the product of the staining intensity and the percentage of cells stained; final scores that ranged from 0 to 300 for each sample were obtained.

\section{Statistical analysis}

SPSS 17.0 statistical software package (SPSS Inc., Chicago, IL, USA) and Stata 12.0 (StataCorp, College Station, TX, USA) were used for all statistical analyses in this study. The frequency distributions were compared by $\chi^{2}$ test. Continuous variables were compared using Student's $t$-test. The primary outcome measure was the length of survival as measured from the time of the original surgery. KaplanMeier survival analysis was used to assess the overall survival (OS), whereas the significant differences between survival curves were determined by the log-rank test. Variables that were found to be significant on univariate analysis at $P \leq 0.05$ were included in multivariate analyses. Cox proportional hazards models were generated for multivariate analyses. All $P$-values $<0.05$ were considered statistically significant by a two-tailed test.

\section{Results}

\section{Immunohistochemical findings}

To evaluate the role of HMGA2 in ccRCC, we first evaluated HMGA2 expression by IHC in 162 specimens from patients with ccRCC. HMGA2 expression was detected in the tumor cell nuclei of $146(90.1 \%)$ cases of ccRCC. Samples from eleven cases showed complete negative staining. Representative immunohistochemical staining results are shown 
in Figure 1. A semiquantitative IHC score was adopted as described in "Materials and methods" section, and the schematic diagram of scoring is shown in Figure 2. The specific staining scores with variable staining intensity in the different specimens ranged from 0 to 290. A median score of 106 was adopted as a cutoff to segregate all samples into either the low expression group (0-106) or the high expression group (107-290).

\section{Correlation between patient characteristics and HMGA2 expression}

The demographic data and clinicopathological characteristics are summarized in Table 1. The present cohort included 58 male and 104 female patients with a mean age of $52.6 \pm 10.6$ years. The mean tumor diameter was $4.5 \pm 2.5 \mathrm{~cm}$, and the TNM staging distribution was as follows: stage I, 42 patients; stage II, 72 patients; stage III, 41 patients; and stage IV, 7 patients. In addition, the Fuhrman staging distribution was as follows: grade I, 44 patients; grade II, 60 patients, grade III, 37 patients, and grade IV, 21 patients. Histological necrosis was observed in $36(22.2 \%)$ patients, and microvascular invasion (MVI) was observed in 26 (16.0\%) patients. Moreover, lymph node metastasis was present in 29 (17.1\%) patients at the time of surgery.

High expression of HMGA2 in the tumor cell nuclei was significantly associated with large tumor size $(P=0.043)$, lymph node metastasis $(P=0.027)$, and higher Fuhrman grade $(P=0.036)$. A borderline significant correlation was observed between HMGA2 nuclear staining and lymph node metastasis $(P=0.027)$. However, no significant correlation was observed

A
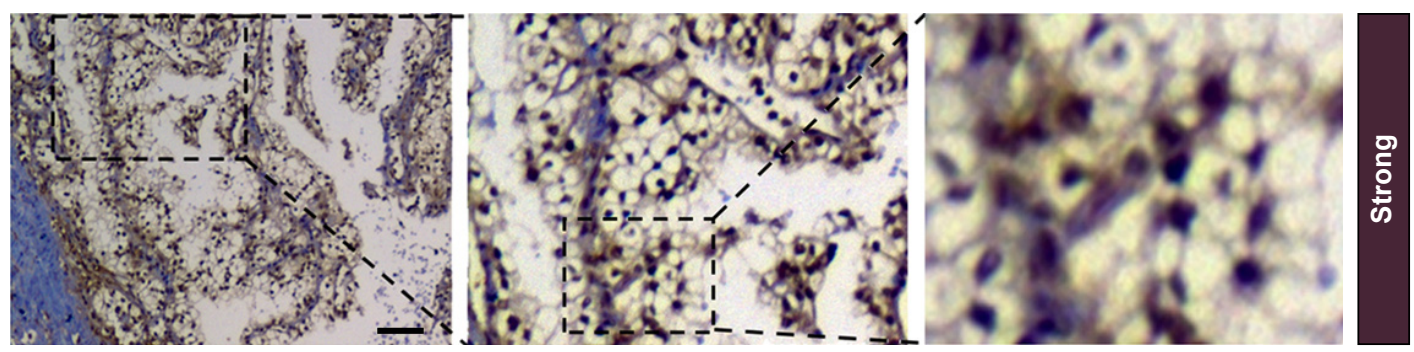

B
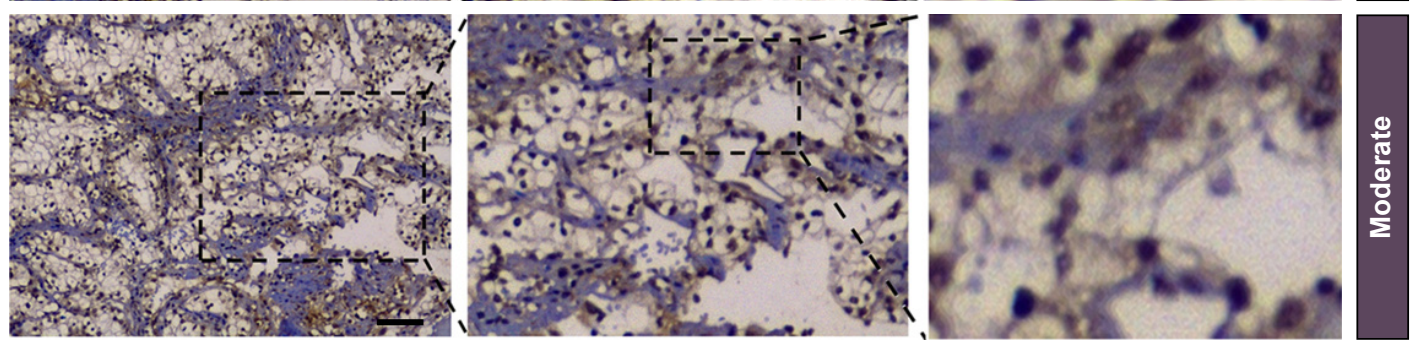

C
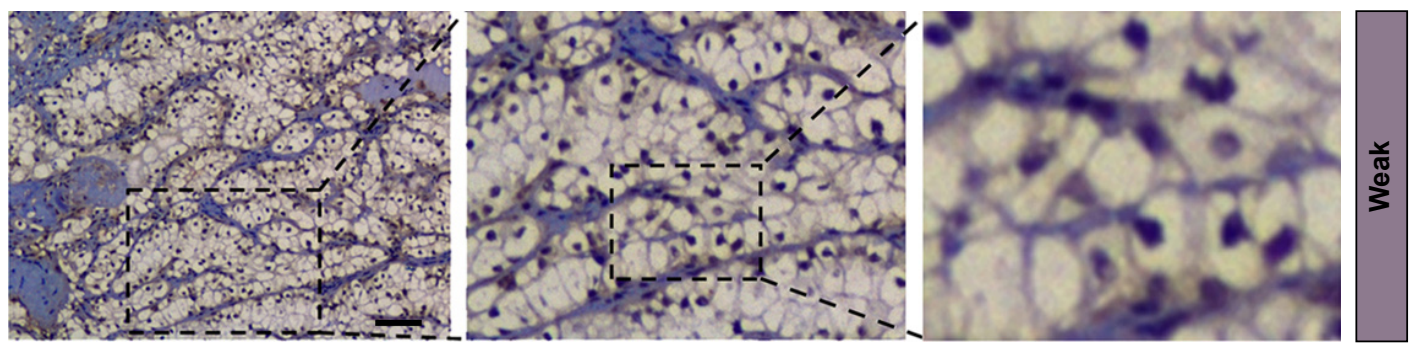

D
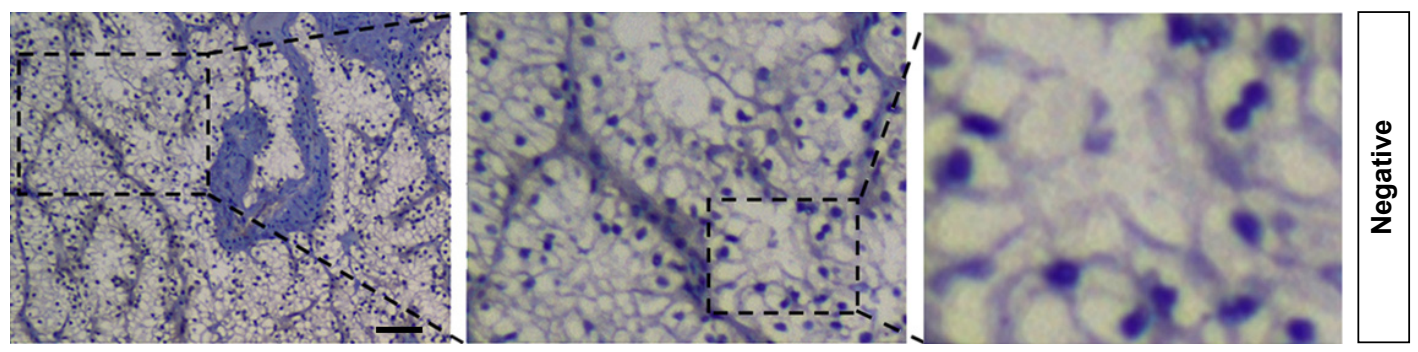

Figure I Typical immunohistochemical staining samples and the corresponding staining intensities of HMGA2 in ccRCC tissues.

Notes: (A) Renal carcinoma tissues showed strong nuclear HMGA2 staining. (B) Renal carcinoma tissues showed moderate nuclear HMGA2 staining. (C) Renal carcinoma tissues showed weak HMGA2 staining. (D) Renal carcinoma tissues showed negative HMGA2 staining. Bars: $50 \mu \mathrm{m}$.

Abbreviations: HMGA2, high-mobility group AT-hook 2; ccRCC, clear cell renal cell carcinoma. 


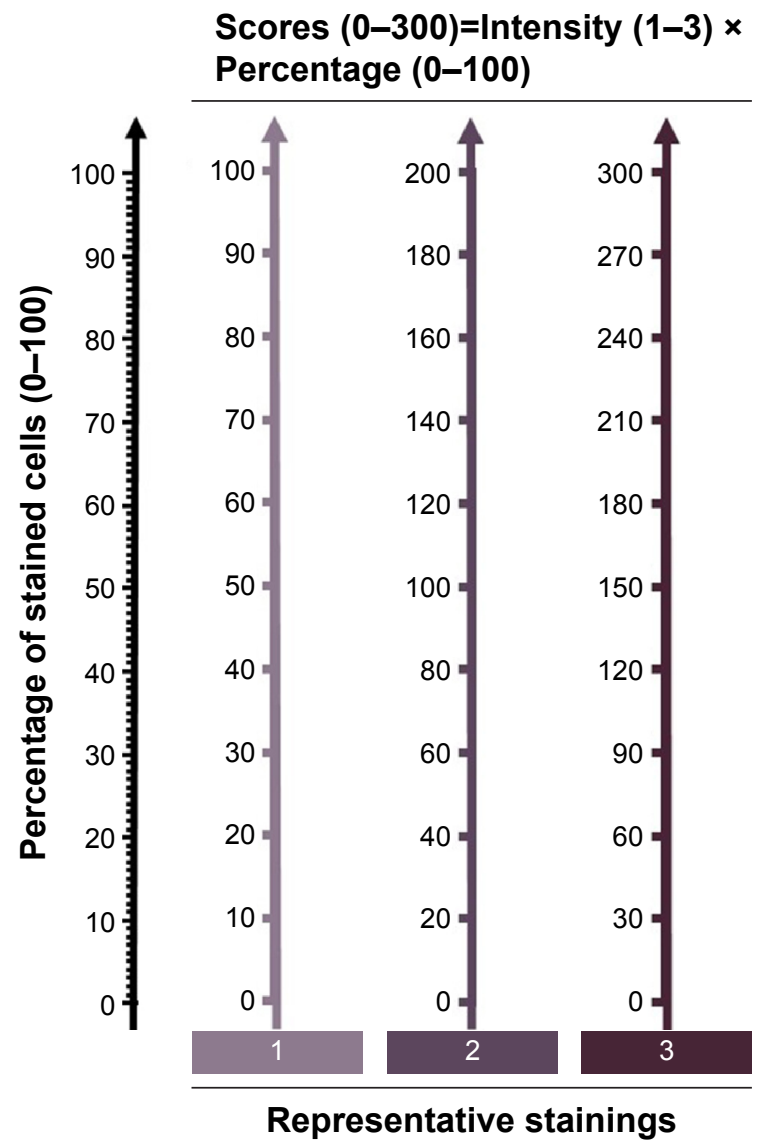

Figure 2 Schematic diagram of scoring.

Notes: The intensity score was obtained based on staining intensity as following: negative, 0; weak, I; moderate, 2; and strong, 3. Meanwhile, the percentage (I-I00) of stained cells was also evaluated in ten randomly selected high-power field. The total score was the product of intensity score and percentage value.

between HMGA2 nuclear staining and patient age, sex, tumor stage, necrosis, or MVI.

\section{Prognostic impact of HMGA2 expression}

Based on the IHC scores for HMGA2, a median score of 106 was adopted as a cutoff to classify all samples into the low expression group $(0-106, \mathrm{n}=86)$ or the high expression group (107-290, n=76). Kaplan-Meier curves were constructed to determine whether HMGA2 expression was correlated with OS in these two groups. Sixty-one patients in the cohort died before the last follow-up. A Kaplan-Meier survival analysis showed that high HMGA2 expression was associated with decreased OS $(P<0.001)$, as shown in Figure 3.

To identify whether HMGA2 expression was an independent prognostic factor in patients with ccRCC, univariate and multivariate analyses were applied for OS. As presented in Table 2, by univariate analysis, we found that HMGA2 expression (hazard ratio $[\mathrm{HR}]=2.815,95 \%$ confidence interval [CI] 1.528-5.186; $P<0.001)$, together with tumor size, pT stage, pN stage, Fuhrman grade, and the presence of MVI,
Table I Correlation between HMGA2 expression and clinicopathological characters in patients with ccRCCC

\begin{tabular}{|c|c|c|c|c|}
\hline \multirow[t]{2}{*}{ Variable } & \multirow{2}{*}{$\begin{array}{l}\text { Patients } \\
\mathrm{n}=162\end{array}$} & \multicolumn{2}{|c|}{ HMGA2 expression } & \multirow[t]{2}{*}{$P$-value } \\
\hline & & Low $(n=86)$ & High (76) & \\
\hline $\begin{array}{l}\text { Age, years } \\
(\text { mean } \pm \text { sd) }\end{array}$ & $52.6 \pm 10.6$ & $51.8 \pm 10.2$ & $53.5 \pm 11.0$ & \\
\hline Sex & & & & 0.795 \\
\hline Female & $58,37.7 \%$ & $30,34.9 \%$ & $28,26.8 \%$ & \\
\hline Male & $104,62.3 \%$ & $56,65.1 \%$ & $48,63.2 \%$ & 0.043 \\
\hline $\begin{array}{l}\text { Tumor size, } \mathrm{cm} \\
\text { (mean } \pm \mathrm{sd})\end{array}$ & $4.5 \pm 2.5$ & $4.1 \pm 2.5$ & $4.9 \pm 2.6$ & \\
\hline pT stage & & & & 0.144 \\
\hline TI & $42,25.9 \%$ & $25,29.1 \%$ & $17,22.4 \%$ & \\
\hline $\mathrm{T} 2$ & $72,44.4 \%$ & $42,48.8 \%$ & $30,39.5 \%$ & \\
\hline T3 & $4 I, 25.3 \%$ & $17,19.8 \%$ & $24,31.6 \%$ & \\
\hline T4 & $7,4.3 \%$ & $2,2.3 \%$ & $5,6.6 \%$ & \\
\hline pN stage ${ }^{a}$ & & & & 0.027 \\
\hline $\mathrm{Nx}-\mathrm{N} 0$ & $133,82.1 \%$ & $76,88.4 \%$ & $57,75.0 \%$ & \\
\hline $\mathrm{NI}$ & $29,17.9 \%$ & $10,11.6 \%$ & $19,25.0 \%$ & \\
\hline Necrosis & & & & 0.239 \\
\hline Absent & $126,77.8 \%$ & $70,81.4 \%$ & $56,73.7 \%$ & \\
\hline Present & $36,22.2 \%$ & $16,18.6 \%$ & $20,26.3 \%$ & \\
\hline Fuhrman grade & & & & 0.036 \\
\hline I & $44,27.2 \%$ & $30,34.9 \%$ & $14,18.4 \%$ & \\
\hline 2 & $60,37.0 \%$ & $34,39.5 \%$ & $26,34.2 \%$ & \\
\hline 3 & $37,22.8 \%$ & $14,16.3 \%$ & $23,30.3 \%$ & \\
\hline 4 & $21,13.0 \%$ & $8,9.3 \%$ & $13,17.1 \%$ & \\
\hline MVI & & & & 0.439 \\
\hline Absent & $136,84.0 \%$ & $74,86.0 \%$ & $62,81.6 \%$ & \\
\hline Present & $26,16.0 \%$ & $12,14.0 \%$ & $14,18.4 \%$ & \\
\hline
\end{tabular}

Notes: ${ }^{\mathrm{a}}$ The $\mathrm{pT}$ stage and $\mathrm{pN}$ stage were classified according to 2010 AJCC TNM classification. 'Student's $t$-test for continuous variables and $\chi^{2}$ test for categorical variables.

Abbreviations: HMGA2, high-mobility group AT-hook 2; ccRCC, clear cell renal cell carcinoma; sd, standard deviation; MVI, microvascular invasion; TNM, tumor node metastasis.

demonstrated a significant association with survival. Further, multivariate Cox regression analysis determined that high HMGA2 expression was a significant negative independent prognostic indicator for patients with ccRCC in an analysis of OS (HR 3.116; 95\% CI: 1.643-5.901; $P<0.001$ ).

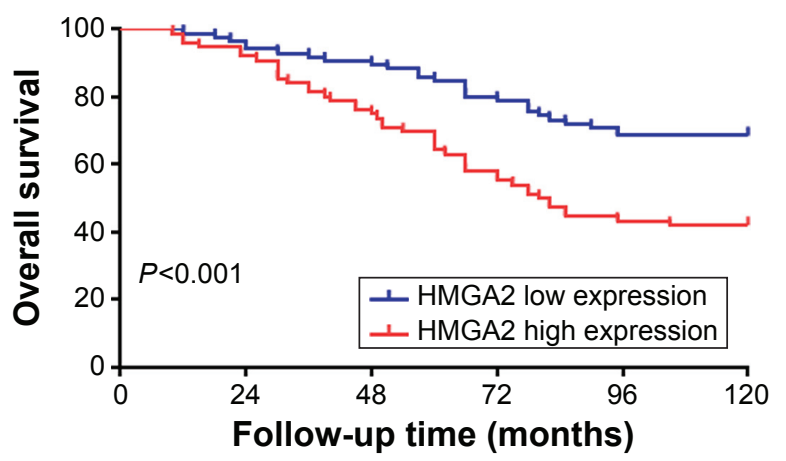

Figure 3 Kaplan-Meier analysis of overall survival of patients with clear cell renal cell carcinoma based on HMGA2 expression.

Note: $P$-value was calculated by log-rank test.

Abbreviation: HMGA2, high-mobility group AT-hook 2. 
Table 2 Univariate and multivariate cox regression analysis identified potential predictors of overall survival in 162 patients with ccRCC

\begin{tabular}{|c|c|c|c|c|c|c|}
\hline \multirow[t]{2}{*}{ Variable } & \multicolumn{3}{|c|}{ Univariate } & \multicolumn{3}{|c|}{ Multivariate } \\
\hline & HR & $95 \% \mathrm{Cl}$ & $P$-value & HR & $95 \% \mathrm{Cl}$ & $P$-value \\
\hline Tumor size, cm & 1.216 & $1.011-1.426$ & 0.038 & 1.172 & $0.966-1.422$ & 0.108 \\
\hline \multicolumn{7}{|l|}{ pT stage } \\
\hline PTI & & Reference & & & & \\
\hline pT2 & 2.107 & $1.126-3.943$ & 0.020 & 2.266 & $1.311-3.917$ & 0.003 \\
\hline pT3 & 3.215 & $1.017-10.163$ & 0.048 & 3.346 & $0.911-12.290$ & 0.069 \\
\hline pT4 & 4.812 & $0.512-45.225$ & 0.169 & 5.011 & $0.719-34.924$ & 0.103 \\
\hline \multicolumn{7}{|l|}{ pN stage } \\
\hline $\mathrm{pNx}+\mathrm{pN} 0$ & & Reference & & & & \\
\hline $\mathrm{pNI}$ & 8.912 & $3.162-25.118$ & $<0.001$ & 6.127 & $2.176-17.254$ & $<0.001$ \\
\hline \multicolumn{7}{|l|}{ Fuhrman grade } \\
\hline 1 & & Reference & & & & \\
\hline 2 & 1.316 & $0.911-1.901$ & 0.143 & 1.255 & $0.95 I-1.656$ & 0.109 \\
\hline 3 & 2.431 & $1.432-4.126$ & $<0.001$ & 2.016 & $1.036-3.923$ & 0.039 \\
\hline 4 & 5.712 & $1.491-21.885$ & 0.011 & 6.112 & $1.636-22.833$ & 0.007 \\
\hline \multicolumn{7}{|l|}{ MVI } \\
\hline Absent & & Reference & & & & \\
\hline Present & 1.423 & $1.025-2.238$ & 0.037 & 1.311 & $0.793-2.167$ & 0.291 \\
\hline \multicolumn{7}{|c|}{ HMGA2 expression } \\
\hline Low expression & & Reference & & & & \\
\hline High expression & 2.815 & $1.528-5.186$ & $<0.001$ & 3.116 & $1.643-5.90 \mid$ & $<0.001$ \\
\hline
\end{tabular}

\section{Expansion of prognostic models for cCRCC patients with the addition of HMGA2 expression}

To further evaluate the value of HMGA2 expression in the improvement of the prognostic prediction for ccRCC, HMGA2 expression was integrated into existing prognostic models including the following scoring systems: TNM staging system, the Mayo Clinic stage, size, grade, and necrosis score, and the University of California Los Angeles Integrated Staging System (UISS). We conducted a Harrell concordance index (C-index) and an Akaike information criteria analysis to investigate the prognostic accuracy of each system. As shown in Table 3, the C-index of the TNM staging system, SSIGN, and UISS was $0.671,0.726$, and 0.711 ,

Table 3 Evaluation the efficacy of prognostic models in predicting the overall survival

\begin{tabular}{ll}
\hline Variable & C-index \\
\hline HMGA2 & 0.641 \\
TNM stage & 0.671 \\
TNM + HMGA2 & 0.719 \\
SSIGN & 0.726 \\
SSIGN + HMGA2 & 0.736 \\
UISS & 0.711 \\
UISS + HMGA2 & 0.723 \\
\hline
\end{tabular}

Abbreviations: C-index, concordance index; HMGA2, high-mobility group AThook 2; TNM, tumor node metastasis; SSIGN, Mayo Clinic stage, size, grade, and necrosis score; UISS, University of California Los Angeles Integrated Staging System. respectively; after the integration of HMGA2 expression into the aforementioned staging systems, the $\mathrm{C}$-index was improved to $0.719,0.736$, and 0.723 , respectively. This suggested that the addition of the HMGA2 expression parameter could improve the prognostic value of all the three models.

\section{Discussion}

Abnormal expression of HMGA2 has been found in many types of malignancies, including colon cancer, breast cancer, pancreatic cancer, ovarian cancer, esophageal cancer, and lung cancer. ${ }^{13}$ It seems that HMGA2 overexpression is associated with a highly malignant phenotype, which results in poor prognosis for patients with these cancers. In the present study, we examined the expression of HMGA2 in ccRCC and evaluated its prognostic value. We confirmed that the expression level of HMGA2 was significantly related to tumor size and Fuhrman grade. Moreover, HMGA2 expression was determined to be a valuable prognostic indicator in patients with ccRCC.

HMGA2, which is a member of the HMGA family, binds to DNA sequences to orchestrate transcriptional activity via the modulation of the chromatin structure. HMGA2 might target different downstream genes to maintain the undifferentiated state of cells during the processes of embryogenesis and tumorigenesis. ${ }^{14}$ Several studies on human cancers have indicated that HMGA2 is important in the survival, proliferation, migration, and metastasis of cancer cells. ${ }^{15-17}$ In addition, 
HMGA2 seems to have oncogenic activities that include the enhancement of self-renewal capability in cancer stem cells and the promotion of tumorigenic capability. ${ }^{18-20}$ As an oncofetal protein, the expression of HMGA2 decreases with the differentiation of tissues, but increases with the dedifferentiation of many malignant tumors. ${ }^{21-23}$ In our present study, we also observed that an increased HMGA2 level was correlated with a higher Fuhrman grade, which suggested a potential role of HMGA2 in tumor dedifferentiation. Consistent with these findings, there were various studies that revealed a positive correlation between HMGA2 expression and tumor grade in a variety of tumor cell types. ${ }^{24-26}$ However, further exploration is needed to clarify the relationship between HMGA2 expression and dedifferentiation. Additionally, our finding that HMGA2 overexpression predicted poor prognosis suggested that HMGA2 overexpression is involved in ccRCC progression, which was consistent with results of previous studies of other types of human cancers.

In the present cohort, the high HMGA2 expression group was more likely to have higher rates of lymph node invasion and vascular invasion than those in the low expression group. Previous studies have reported a strong correlation between HMGA2 expression and tumor cell invasiveness. ${ }^{27-29}$ The ectopic expression of HMGA2 in epithelial cells induces epithelial-mesenchymal transition (EMT), which has been implicated in the acquisition of metastatic characteristics in tumor cells. ${ }^{30}$ Notably, several studies have reported that HMGA2 plays a important role in EMT during diabetic nephropathy, ${ }^{31,32}$ indicating a potential role of HMGA2 in EMT of renal cancer cells. This may occur through activation of the Wnt/ $\beta$-catenin pathway, ${ }^{28-30,33}$ or through the regulation of the transcription factors involved in EMT including Snail, Slug, and Twist. ${ }^{30,31}$ Besides, HMGA2 can also induce EMT through an interaction via the TGF $\beta$ signaling pathway that has been strongly implicated in EMT induction for a number of cell types both in vitro and in vivo..$^{30,34,35}$ In this respect, HMGA2 plays an important role in tumor invasiveness and metastasis, which supports our findings that HMGA2 overexpression is associated with clinicopathological factors such as lymph node metastasis and MVI.

Although HMGA2 is gradually being recognized as a prognostic biomarker in many malignancies, the prognostic value of HMGA2 in ccRCC remains unclear. In the present study, we found that a high level of HMGA2 expression in ccRCC patients was significantly associated with a poor prognosis. The multivariate analysis showed that HMGA2 expression was an independent prognostic factor for survival. Furthermore, we determined that the addition of HMGA2 expression to existing prognostic prediction models, such as the TNM staging system, could improve their prediction power. Therefore, the detection of the HMGA2 level in ccRCC tissues after surgery might be helpful to further predict the prognosis of those patients. However, this study still has several limitations. The sample size of our cohort was not large enough, and the study was retrospective, which means that our study is not sufficient to achieve greater reliability. Finally, further research on the molecular biological mechanism of HMGA2 in ccRCC is warranted.

\section{Conclusion}

In conclusion, our study revealed that HMGA2 expression is an independent prognostic factor for OS in patients with ccRCC. The abnormal expression of HMGA2 may play a role in the carcinogenesis of ccRCC. These results provide a basis for further, more detailed studies on the roles of HMGA2 in ccRCC.

\section{Acknowledgment}

This study was supported by the Natural Science Foundation of China (81470977).

\section{Disclosure}

The authors report no conflicts of interest in this work.

\section{References}

1. Srigley JR, Delahunt B, Eble JN, et al. The International Society of Urological Pathology (ISUP) Vancouver Classification of Renal Neoplasia. Am J Surg Pathol. 2013;37(10):1469-1489.

2. Siegel RL, Miller KD, Jemal A. Cancer statistics, 2015. CA Cancer J Clin. 2015;65(1):5-29.

3. Battaglia M, Lucarelli G. The role of renal surgery in the era of targeted therapy: the urologist's perspective. Urologia. 2015;82(3):137-138.

4. Sun M, Shariat SF, Cheng C, et al. Prognostic factors and predictive models in renal cell carcinoma: a contemporary review. Eur Urol. 2011; 60(4):644-661

5. Kim HL, Halabi S, Li P, et al. A molecular model for predicting overall survival in patients with metastatic clear cell renal carcinoma: results from CALGB 90206 (alliance). EBioMedicine. 2015;2(11):1814-1820.

6. Chen F, Zhang Y, Şenbabaoğlu Y, et al. Multilevel Genomics-Based Taxonomy of Renal Cell Carcinoma. Cell Rep. 2016;14(10):2476-2489.

7. Wang J, Liu Y, Yang Y, et al. High expression of galectin-7 associates with poor overall survival in patients with non-metastatic clear-cell renal cell carcinoma. Oncotarget. Epub 2016 May 31.

8. Fu H, Liu Y, Xu L, et al. Low expression of mucin-4 predicts poor prognosis in patients with clear-cell renal cell carcinoma. Medicine (Baltimore). 2016;95(17):e3225.

9. Johnson KR, Lehn DA, Reeves R. Alternative processing of mRNAs encoding mammalian chromosomal high-mobility-group proteins HMG-I and HMG-Y. Mol Cell Biol. 1989;9(5):2114-2123.

10. Ozturk N, Singh I, Mehta A, Braun T, Barreto G. HMGA proteins as modulators of chromatin structure during transcriptional activation. Front Cell Dev Biol. 2014;2:5.

11. Chiappetta G, Avantaggiato V, Visconti R, et al. High level expression of the HMGI (Y) gene during embryonic development. Oncogene. 1996;13(11):2439-2446. 
12. Takaha N, Sowa Y, Takeuchi I, Hongo F, Kawauchi A, Miki T. Expression and role of HMGA1 in renal cell carcinoma. J Urol. 2012;187(6): 2215-2222.

13. Pallante P, Sepe R, Puca F, Fusco A. High mobility group a proteins as tumor markers. Front Med (Lausanne). 2015;2:15.

14. Yu KR, Shin JH, Kim JJ, et al. Rapid and efficient direct conversion of human adult somatic cells into neural stem cells by HMGA2/let-7b. Cell Rep. Epub 2015 Jan 15.

15. Shi Z, Wu D, Tang R, et al. Silencing of HMGA2 promotes apoptosis and inhibits migration and invasion of prostate cancer cells. J Biosci. 2016;41(2):229-236.

16. Sun M, Song CX, Huang H, et al. HMGA2/TET1/HOXA9 signaling pathway regulates breast cancer growth and metastasis. Proc Natl Acad Sci U S A. 2013;110(24):9920-9925.

17. Natarajan S, Hombach-Klonisch S, Droge P, Klonisch T. HMGA2 inhibits apoptosis through interaction with ATR-CHK1 signaling complex in human cancer cells. Neoplasia. 2013;15(3):263-280.

18. Kaur H, Ali SZ, Huey L, et al. The transcriptional modulator HMGA2 promotes stemness and tumorigenicity in glioblastoma. Cancer Lett. 2016;377(1):55-64.

19. Ikeda K, Ogawa K, Takeishi Y. The role of HMGA2 in the proliferation and expansion of a hematopoietic cell in myeloproliferative neoplasms. Fukushima J Med Sci. 2012;58(2):91-100.

20. Madison BB, Jeganathan AN, Mizuno R, et al. Let-7 represses carcinogenesis and a stem cell phenotype in the intestine via regulation of Hmga2. PLoS Genet. 2015;11(8):e1005408.

21. Piscuoglio S, Zlobec I, Pallante P, et al. HMGA1 and HMGA2 protein expression correlates with advanced tumour grade and lymph node metastasis in pancreatic adenocarcinoma. Histopathology. 2012; 60(3):397-404.

22. Mu G, Liu H, Zhou F, et al. Correlation of overexpression of HMGA1 and HMGA2 with poor tumor differentiation, invasion, and proliferation associated with let-7 down-regulation in retinoblastomas. Hum Pathol. 2010;41(4):493-502.

23. Parameswaran S, Xia X, Hegde G, Ahmad I. Hmga2 regulates self-renewal of retinal progenitors. Development. 2014;141(21):4087-4097.
24. Rogalla P, Drechsler K, Kazmierczak B, Rippe V, Bonk U, Bullerdiek J. Expression of HMGI-C, a member of the high mobility group protein family, in a subset of breast cancers: relationship to histologic grade. Mol Carcinog. 1997;19(3):153-156.

25. Wang X, Liu X, Li AY, et al. Overexpression of HMGA2 promotes metastasis and impacts survival of colorectal cancers. Clin Cancer Res. 2011;17(8):2570-2580.

26. Miyazawa J, Mitoro A, Kawashiri S, Chada KK, Imai K. Expression of mesenchyme-specific gene HMGA2 in squamous cell carcinomas of the oral cavity. Cancer Res. 2004;64(6):2024-2029.

27. Wu J, Wang Y, Xu X, et al. Transcriptional activation of FN1 and IL11 by HMGA2 promotes the malignant behavior of colorectal cancer. Carcinogenesis. 2016;37(5):511-521.

28. Yang E, Cisowski J, Nguyen N, et al. Dysregulated protease activated receptor 1 (PAR1) promotes metastatic phenotype in breast cancer through HMGA2. Oncogene. 2016;35(12):1529-1540.

29. Xia YY, Yin L, Jiang N, et al. Downregulating HMGA2 attenuates epithelial-mesenchymal transition-induced invasion and migration in nasopharyngeal cancer cells. Biochem Biophys Res Commun. 2015; 463(3):357-363.

30. Morishita A, Zaidi MR, Mitoro A, et al. HMGA2 is a driver of tumor metastasis. Cancer Res. 2013;73(14):4289-4299.

31. Bai YH, Wang JP, Yang M, Zeng Y, Jiang HY. SiRNA-HMGA2 weakened AGEs-induced epithelial-to-mesenchymal transition in tubular epithelial cells. Biochem Biophys Res Commun. 2015;457(4):730-735.

32. Liu H, Wang X, Liu S, et al. Effects and mechanism of miR-23b on glucose-mediated epithelial-to-mesenchymal transition in diabetic nephropathy. Int J Biochem Cell Biol. 2016;70:149-160.

33. Zha L, Zhang J, Tang W, et al. HMGA2 elicits EMT by activating the Wnt/beta-catenin pathway in gastric cancer. Dig Dis Sci. 2013;58(3): 724-733.

34. Lee AW, Ma BB, Ng WT, Chan AT. Management of Nasopharyngeal Carcinoma: Current Practice and Future Perspective. J Clin Oncol. 2015; 33(29):3356-3364.

35. Chua ML, Wee JT, Hui EP, Chan AT. Nasopharyngeal carcinoma Lancet. 2016;387(10022):1012-1024
OncoTargets and Therapy

\section{Publish your work in this journal}

OncoTargets and Therapy is an international, peer-reviewed, open access journal focusing on the pathological basis of all cancers, potential targets for therapy and treatment protocols employed to improve the management of cancer patients. The journal also focuses on the impact of management programs and new therapeutic agents and protocols on

\section{Dovepress}

patient perspectives such as quality of life, adherence and satisfaction. The manuscript management system is completely online and includes a very quick and fair peer-review system, which is all easy to use. Visit http://www.dovepress.com/testimonials.php to read real quotes from published authors. 